\title{
Diagnostic précoce de gravidité chez la vache grâce à l'observation de l'éjection du lait induite par l'ocytocine lutéale
}

\author{
J Labussière 1, JF Combaud 1, FA de la Chevalerie 1, \\ D André 2 , JL Touzé 2 , J Cochaud 3 \\ ${ }^{1}$ INRA, laboratoire de la traite, 65 rue de St-Brieuc, 35042 Rennes Cedex; \\ 2 INRA, station de physiologie de la reproduction, Nouzilly, 37380 Monnaie; \\ ${ }^{3}$ INRA, domaine de Bressonvilliers, Leudeville, 91630 Marolles-en-Hurepoix, France
}

(Reçu le 29 novembre 1991; accepté le 10 mars 1992)

\begin{abstract}
Résumé - Le diagnostic de gravidité décrit dans cet article repose sur l'observation de l'éjection lactée qui, en cas de maintien du corps jaune, résulte de la libération d'ocytocine lutéale induite par l'administration intraveineuse d'une dose non lutéolytique de PGF2 $\alpha$. Les contrôles ont été réalisés $3 \mathrm{~h}$ avant la traite du soir chez 410 vaches en lactation, 18-22 j après leur insémination (moyenne : $19,95 \mathrm{j}$, écart type 0,75 ). Une canule est placée dans le trayon avant gauche; lorsque le lait citernal cesse de couler, on injecte dans la veine jugulaire (ou dans la veine mammaire abdominale) $256 \mu \mathrm{g}$ de Dinolytic, un analogue de la prostaglandine F2a. Si un corps jaune est présent, on assiste, $86 \pm$ 35 s plus tard, à l'écoulement du lait alvéolaire (1 $276 \pm 570 \mathrm{ml})$; on peut alors présumer un début de gravidité. Si le corps jaune n'est plus fonctionnel, il n'y a pas de nouvel écoulement; on peut conclure que la vache n'est pas gravide. L'exactitude des résultats positifs $(72,3 \%)$ est supérieure $(P<0,05)$ à celle obtenue avec le dosage radio-immunologique de la progestérone plasmatique qui a servi de méthode de référence $(68,6 \%)$. Celle des résultats négatifs est, par contre, inférieure $(93,5 \%$ vs $100 \%)(P<0,05)$, car, parmi les 195 vaches gravides jusqu'à terme, 10 n'ont pas répondu au test d'éjection du lait. Malgré ces réserves, ce test présente l'avantage d'être peu coûteux, rapide (environ $5 \mathrm{~min}$ ) et facile à interpréter immédiatement (le lait coule ou ne coule pas). Enfin, il peut être utilisé précocement, puisque, dès le $20^{\circ}$ jour après la tentative de fécondation, la fiabilité de prédiction des vaches qui ne vêleront pas $(67,0 \%)$ est supérieure à celle obtenue à partir de la progestéronémie $(58,6 \%, P<0,05)$. Ainsi, sans perdre 3 semaines, il est envisageable de réinséminer immédiatement les femelles non gravides selon ce test, afin de réduire la durée des périodes infertiles.
\end{abstract}

diagnostic de gravidité / éjection lactée / vache / ocytocine lutéale / prostaglandine

Summary - Early diagnosis of pregnancy in cows as assessed by milk ejection induced by luteal oxytocin. The diagnosis of pregnancy described in this article is based on the observation of milk ejection which, in the case of corpus luteum maintenance, results from the release of luteal oxytocin induced by intravenous administration of a non luteolytic dose of PGF2 $\alpha$. The tests were performed in 410 lactating cows, 18-22 days after insemination (mean 19.95 days, SD 0.75), $3 \mathrm{~h}$ prior to the evening milking. A cannula was placed in the left fore-teat; when the cisternal milk flow 
ceased, a small dose of a prostaglandin F2 $\alpha$ analogue (256 $\mu \mathrm{g}$ Dinolytic) was injected. If a corpus luteum was present, the alveolar milk flow (1 $276 \pm 570 \mathrm{ml}$ ) started $86 \pm 35 \mathrm{~s}$ later and pregnancy could be presumed. If the corpus luteum was no longer functional, the milk flow did not start again and the cow was considered not pregnant. The accuracy of positive results, ie $72.3 \%$, significaritly exceeded that obtained by radioimmunoassay of plasma progesterone used as a reference method, ie $68.6 \%$ $(P<0.05)$. By contrast, the accuracy of negative results was lower, ie 93.5 vs $100 \%,(P<0.05)$ : of the 195 pregnant cows which proceeded to term, 10 did not respond to the milk ejection test. In spite of these reserves, the test has the advantage of being inexpensive, rapid (about $5 \mathrm{~min}$ ) and easy to interpret, ie either the milk flows or it does not. In addition, it may be used early, because from day 20 after $A /$ the reliability of prediction of non pregnant cows (67.0\%) was significantly higher when compared to the use of progesterone radioimmunoassay $(58.6 \%)(\mathrm{P}<0.05)$. With such a test, non pregnant cows can be reinseminated immediately and not only after a 3-week period. This may contribute to reducing the length of the infertile period.

\section{pregnancy diagnosis / milk ejection / cow / luteal oxytocin / prostaglandin}

\section{INTRODUCTION}

Pour respecter un intervalle entre vêlages de $365 \mathrm{j}$, l'éleveur doit savoir le plus rapidement possible après l'insémination, si une vache est gravide. L'observation échographique de l'embryon ou l'apparition dans le sang maternel de sécrétions trophoblastiques (Pregnant Specific Protein B: Sasser et al, 1986) sont des signes directs de gravidité chez la vache. Toutefois, l'exactitude de la méthode échographique n'est satisfaisante qu'après le $35^{\mathrm{e}} \mathrm{j}$ de vie fœtale (Tainturier et al, 1983; Chaffaux et al, 1988) et ce n'est qu'à partir du $30^{e}$ j que le test PSPB offre suffisamment de garantie (Humblot et al, 1988) dans la mesure où chez les multipares, il ne subsiste pas de reliquats de PSPB de la gestation précédente qui interdisent son emploi avant le $100 \mathrm{e}$ j post-partum (Humblot, 1991). Les diagnostics plus précoces ne sont donc pas basés sur la recherche directe de l'embryon, mais sur celle du maintien du corps jaune au stade où, a contrario, il y a déjà lutéolyse chez les femelles cycliques ou non gravides. C'est la progestérone qui, jusqu'à maintenant, a été l'unique “révélateur» de l'activité lutéale par les variations de sa concentration dans le plasma sanguin (Terqui et André, 1982; Humblot et Thibier, 1984; Kishimoto et al, 1987) et dans le lait (Laing et Heap, 1973; Heap et al, 1976; Hoffman et al, 1976; Cox et al, 1978; Booth et al, 1979; Thibier, 1980; Laitinen et al, 1985; Sauer et al, 1986; Stanley et al, 1986; Van de Wiel et Koops, 1986).

Les tests immuno-enzymatiques (EIA) sur des échantillons de lait offrent l'avantage d'une lecture directe à la ferme par rapport aux dosages radioimmunologiques (sanguins ou lactés) qui imposent des délais d'acheminement pour l'envoi des tubes au laboratoire et qui interdisent les réinséminations immédiates dès la fin des cycles jugés infertiles. Ce n'est, en effet, qu'à partir du 21 e j après l'insémination, que la détection de la progestérone par RIA ou EIA permet d'atteindre des pourcentages d'exactitude satisfaisants pour les diagnostics positifs (70-75\%) et très proches de $100 \%$ pour les diagnostics négatifs (Humblot et al, 1987). Par ailleurs, malgré leur apparente simplicité, les kits EIA peuvent parfois exiger des temps de réaction de 20-40 min pour que la lecture soit interprétable (Tainturier et al, 1988). 
En outre, la plupart d'entre eux sont onéreux (Mialot, 1988), ce qui freine leur utilisation systématique dans de nombreux troupeaux.

Ces raisons nous ont conduit à essayer de repérer les corps jaunes de début de gravidité non plus par leur sécrétion de progestérone, mais par celle de l'ocytocine. En effet, il est maintenant admis que les corps jaune peuvent également synthétiser ce peptide ( $c f$ revue de Wathes et al, 1986) dont les concentrations tissulaires diminuent plus précocement que celles de progestérone en cas d'échec de fécondation (Wathes et al, 1984; Ivell et al, 1985).

Nous avons montré que l'injection intraveineuse de $256 \mu \mathrm{g}$ de prostaglandine $\mathrm{F} 2 \alpha$ n'entraîne aucun risque de lutéolyse (Labussière et al, 1988) mais provoque une décharge d'ocytocine lutéale (Labussière et al, 1990) et une éjection lactée qui peut être mise en évidence par enregistremen$\mathrm{t} E x_{d N}$ de la pression intramammaire ou par mesure du lait qui s'écoule d'un trayon canulé (Labussière et al, 1982).

Dans ces conditions et sur des effectifs limités, il nous est apparu possible de discriminer les vaches gravides et cycliques dès le $19^{\mathrm{e}}$ ou $20^{\mathrm{e}} \mathrm{j}$ après l'insémination. Nous avons donc souhaité tester l'exactitude et la fiabilité de cette nouvelle méthode de diagnostic sur un grand nombre d'animaux.

\section{MATÉRIEL ET MÉTHODES}

Les résultats de 410 inséminations ont été évalués dans 3 troupeaux de vaches laitières $(207$, 144 et 59 animaux) à l'aide de 2 méthodes :

- la détection de l'écoulement lacté après administration intraveineuse de PGF2 $\alpha$;

- le dosage radio-immunologique de progestérone dans un échantillon sanguin prélevé $2-3 \mathrm{~h}$ avant le test d'éjection du lait.

Les contrôles sont réalisés en moyenne 19,95 j après l'insémination (8, 104, 208, 80 et
10 cas respectivement aux $18^{\ominus}, 19^{e}, 20^{\circ}, 21^{\theta}$ et $\left.22^{\mathrm{e}} \mathrm{j}\right)$. Les réponses des 2 tests sont confrontées à l'observation des retours en chaleurs (à $21 \pm 2 \mathrm{j}$ post-oestrus ou au-delà du $24^{\circ} \mathrm{j}$ ) et à celle des vêlages (ou des avortements tardifs après 250 j de gravidité). Ceci a permis de calculer :

$$
\begin{aligned}
& E x_{d P}=\frac{V_{P v}}{V_{P}} \times 100 \\
& E x_{d N}=\frac{V_{N m}}{V_{N}} \times 100 \\
& F_{d P}=\frac{V_{P V}}{V_{v}} \times 100 \\
& F_{d N}=\frac{V_{N n}}{V_{n}} \times 100
\end{aligned}
$$

avec $E x_{d P}=$ exactitude des diagnostics positifs; $E x_{d N}=$ exactitude des diagnostics négatifs; $F_{d P}$ = fiabilité des diagnostics positifs; $F_{d N}=$ fiabilité des diagnostics négatifs; $V_{P y}=$ vaches positives ayant vêlé; $V_{N m}=$ vaches négatives n'ayant pas vêlé; $V_{P}=$ nombre de vaches positives; $V_{N}=$ nombre de vaches négatives; $V_{v}=$ nombre de vaches ayant vêlé; $V_{n}=$ nombre de vaches n'ayant pas vêlé.

\section{Méthode de diagnostic basée sur l'éjection du lait}

Deux à trois heures avant la traite du soir, une canule est placée dans le trayon avant gauche afin d'évacuer le lait citernal dont le volume et le temps d'écoulement sont mesurés.

La canule est une sonde métallique stérile de $10 \mathrm{~cm}$ de long (cône international $6 \%$ à verrou de marque Crépin); l'extrémité du trayon est désinfectée avec 2 compresses imbibées de savon liquide iodé (Vétédine de Vétoquinol) et d'alcool à $90^{\circ}$. Ce nettoyage doit être réalisé avec délicatesse pour éviter toute décharge d'ocytocine posthypophysaire.

Lorsque la citerne est vide, on injecte dans la veine mammaire abdominale (troupeau 1) ou dans la veine jugulaire (troupeaux 2 et 3 ), une 
faible dose, non lutéolytique (Labussière et al, 1988), d'un sel de trométhamine de PGF2 $\alpha$ (256 $\mathrm{\mu g}$ de Dinolytic-Upjohn) dilué dans $2 \mathrm{ml}$ de $\mathrm{NaCl} 9 \%$.

Au cours des 4 min qui suivent, 2 cas peuvent se présenter; ils ont servi de base au diagnostic de gestation :

- observation d'un nouvel écoulement qui correspond à l'expulsion du lait alvéolaire sous l'effet de la libération d'ocytocine lutéale. Le résultat est positif et la vache est présumée gravide. Les caractéristiques de ce flux (volume, durée, et temps de latence depuis l'injection) ont également été mesurées;

- pas de nouvel écoulement; ceci traduit l'absence de corps jaune, le résultat est négatif et la vache est diagnostiquée non gravide.

\section{Méthode de diagnostic basée sur la recherche de la progestérone}

Deux à trois heures avant le test d'éjection du lait, $10 \mathrm{ml}$ de sang sont prélevés (vacutainer hépariné), dans la veine de la queue (troupeaux 1 et 2) ou dans la veine jugulaire (troupeau 3). L'échantillon est immédiatement centrifugé $\left(15 \mathrm{~min}\right.$ à $3000 \mathrm{tr} / \mathrm{min}$ à $4^{\circ} \mathrm{C}$ ) et le surnageant congelé pour être soumis au dosage semiquantitatif (méthode rapide) et quantifatif de la progestérone.

\section{La technique semi-quantitative}

Décrite par Terqui et Thimonier (1974) et modifiée par Tamboura (1983), elle sert pour discriminer les résultats positifs et négatifs. L'interprétation des analyses, en terme de diagnostic de non-gestation ou de gestation, s'opère à l'aide de 2 échantillons aux concentrations discriminantes $(0,8 \mathrm{ng} / \mathrm{ml}$ et $1,5 \mathrm{ng} / \mathrm{ml})$ régulièrement introduits dans les échantillons inconnus. Audessus de $1,5 \mathrm{ng} / \mathrm{ml}$, la vache est présumée pleine alors qu'en dessous de $0,8 \mathrm{ng} / \mathrm{ml}$, elle est considérée comme non gravide. Entre ces 2 valeurs, les résultats sont classés douteux.

\section{La technique quantitative}

Elle est adaptée de celle proposée par Saumande et al (1985) et permet de mieux appré- hender la signification des valeurs de progestéronémie en fonction des caractéristiques d'éjection. Les variations intradosages ont été de $6,2 \%$ pour un niveau moyen de $1,3 \mathrm{ng} / \mathrm{ml}(n=$ 18 ) et de $11,5 \%$ pour un niveau moyen de 5,0 $\mathrm{ng} / \mathrm{ml}(n=18)$. Les variations interdosages observées au cours de l'expérience furent de $5,9 \%$ pour un niveau moyen de $1,4 \mathrm{ng} / \mathrm{ml}(n=137)$ et de $6,3 \%$ pour un niveau moyen de $4,6 \mathrm{ng} / \mathrm{ml}$ $(n=137)$.

\section{Analyses statistiques}

Les différences entre les coefficients d'exactitude des 2 diagnostics de gravidité ont été testées selon la méthode de comparaison de caractères qualitatifs à 2 classes; celles entre les coefficients de fiabilité reposent sur l'analyse des paires de résultats discordants (Schwartz, 1963). Des analyses de variance accompagnées de comparaisons multiples de Scheffe ont été réalisées sur les paramètres quantitatifs caractérisant les réponses de progestérone et d'éjection du lait.

\section{RÉSULTATS}

\section{Caractéristiques des réponses (tableau I)}

1) Les 410 inséminations conduisent à :

- cent dix-sept retours normaux en œstrus entre 19 et $23 \mathrm{j}$ (moyenne el écart type $21,1 \pm 1,2)$;

- quatre-vingt-dix-huit retours en œstrus tardifs entre 23 et $250 \mathrm{j}(54,2 \pm 33,5)$;

- cent quatre-vingt-quinze vêlages à 280,4 $\pm 6,2 \mathrm{j}$.

2) II faut en moyenne $112 \mathrm{~s}$ (extrêmes 5 à $570 \mathrm{~s})$ pour que les $231 \mathrm{ml}$ de lait citernal (2 à $1620 \mathrm{ml}$ ) s'écoulent par la canule placée dans le canal du trayon. Sans que la différence soit significative, cette fraction citernale a tendance à être plus volumineuse $(269 \mathrm{ml})$ chez les 117 vaches qui re- 


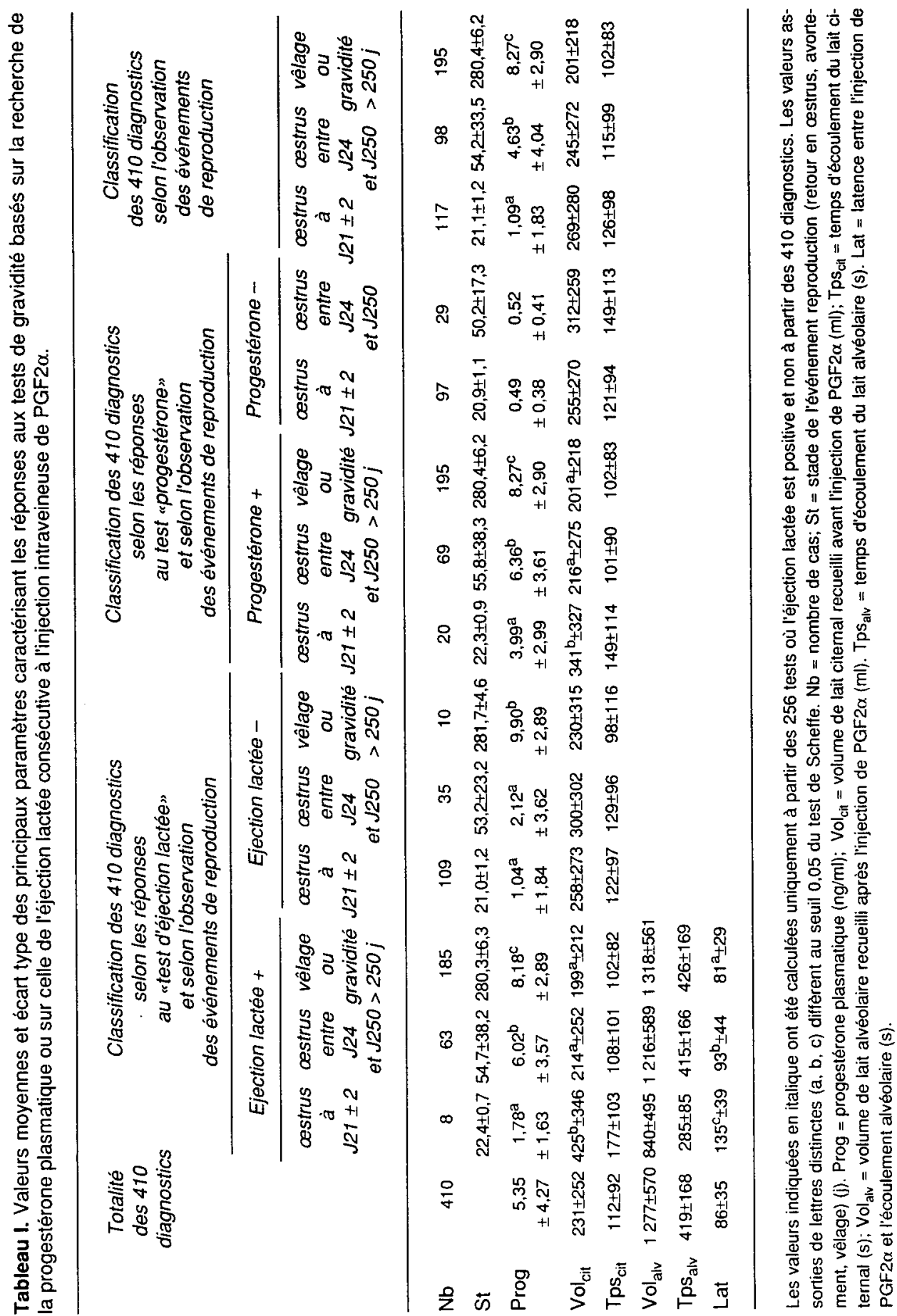


viennent en chaleur à $21 \pm 2$ jours que chez les 195 futures parturientes $(201 \mathrm{ml})$.

3) II faut attendre en moyenne $86 \mathrm{~s}$ (30 à $240 \mathrm{~s})$ entre l'administration intraveineuse de Dinolytic et le début de l'expulsion des $1277 \mathrm{ml}$ de lait alvéolaire (80 à $3600 \mathrm{ml}$ ); ce délai semble plus court et le volume évacué plus abondant, lorsque le corps jaune est bien «installé» et doit persister longtemps. En effet, on observe respectivement l'éjection de 1318,1216 et 840 $\mathrm{ml}$ (NS) après une latence de 81,93 et 135 s $(P<0,05)$ selon que les vaches auront une gravidité supérieure à $250 \mathrm{j}$, un retour en chaleur tardif (J24 à J250) ou normal (J21 \pm 2$)$.

Lorsque le volume du lait alvéolaire est pris en compte comme $2^{e}$ critère de diagnostic du succès ou de l'échec de l'insémination, en complément de la concentration plasmatique de progestérone, il apparaît que :

- les 195 vaches qui restent gravides jusqu'à terme, présentent au moment du test à la fois une progestéronémie élevée (aucun vêlage observé en-dessous de 2,4 $\mathrm{ng} / \mathrm{ml}$ et seulement $7,5 \%$ en-dessous de $4,8 \mathrm{ng} / \mathrm{ml}$ ) et aussi une aptitude à éjecter un volume de lait important sous l'effet de PGF2 $\alpha(97 \%$ des futures parturientes se situent au-dessus de $1200 \mathrm{ml}$ et de 4,8 $\mathrm{ng} / \mathrm{ml})$;

- les 117 femelles revenant en chaleur précocement avant 23 j ont des caractéristiques inverses : $84,6 \%$ n'atteignent pas $400 \mathrm{ml}$ et $2,4 \mathrm{ng} / \mathrm{ml}$ et 109 sur $117(93,7 \%)$ n'ont aucun écoulement, bien que 11 d'entre elles présentent encore une progestéronémie résiduelle supérieure à 2,4 $\mathrm{ng} / \mathrm{ml}$;

- les 98 cas intermédiaires à œstrus tardif (après $24 \mathrm{j}$ ), ou à avortement avant $250 \mathrm{j}$, sont plus difficiles à identifier. Leur taux de progestérone et leur volume alvéolaire sont le plus souvent proches de ceux des vaches dont la gravidité est confirmée par une mise-bas, mais, pour 28 vaches, il n'y a eu aucun écoulement et la progestéronomie est restée inférieure à $2,4 \mathrm{ng} / \mathrm{ml}$.

\section{Exactitude et fiabilité des diagnostics}

L'exactitude des résultats négatifs est de $100 \%$ avec la progestérone (tableau II); elle est significativement supérieure $(P<$ 0,01 ) à celle obtenue avec le test d'éjection $(93,5 \%)$. Le stade auquel sont effectués les diagnostics influence peu les valeurs de ces coefficients (fig 1). L'exactitude des résultats positifs est, par contre, significativement améliorée $(P<0,01)$ lorsqu'on se réfère à l'évacuation du lait $(72,3 \%)$ plutôt qu'aux indications de progestéronémie $(68,2 \%)$. La figure 1 montre que l'exactitude s'accroît avec le délai entre l'insémination et la date du diagnostic; dès le $20^{\mathrm{e}} \mathrm{j}$, elle atteint $73,2 \%$ pour le test d'écoulement.

La fiabilité des résultats positifs avec la progestérone est de $100 \%$ car les 195 vaches ayant vêlé avaient toutes été présumées gravides à l'aide de ce test (tableau II). L'observation de l'écoulement du lait est significativement moins efficace $(P<0,01)$ puisqu'elle ne permet d'en détecter que $185(94,9 \%)$. Le stade auquel sont effectués les diagnostics influence peu les valeurs de ces coefficients (fig 2).

Par contre, l'absence d'éjection lactée est un meilleur indicateur que l'absence de progestérone pour connaître précocement les femelles qui ne vêleront pas. La fiabilité des résultats négatifs est respectivement de $67,0 \%$ et $58,6 \%(P<0,01)$ et dès le $19 \mathrm{e}$ j, on atteint déjà $69,3 \%$ avec la canulation du trayon (fig 2).

Cette méthode est donc plus efficace que le dosage de la progestérone pour connaître précocement les femelles vides 


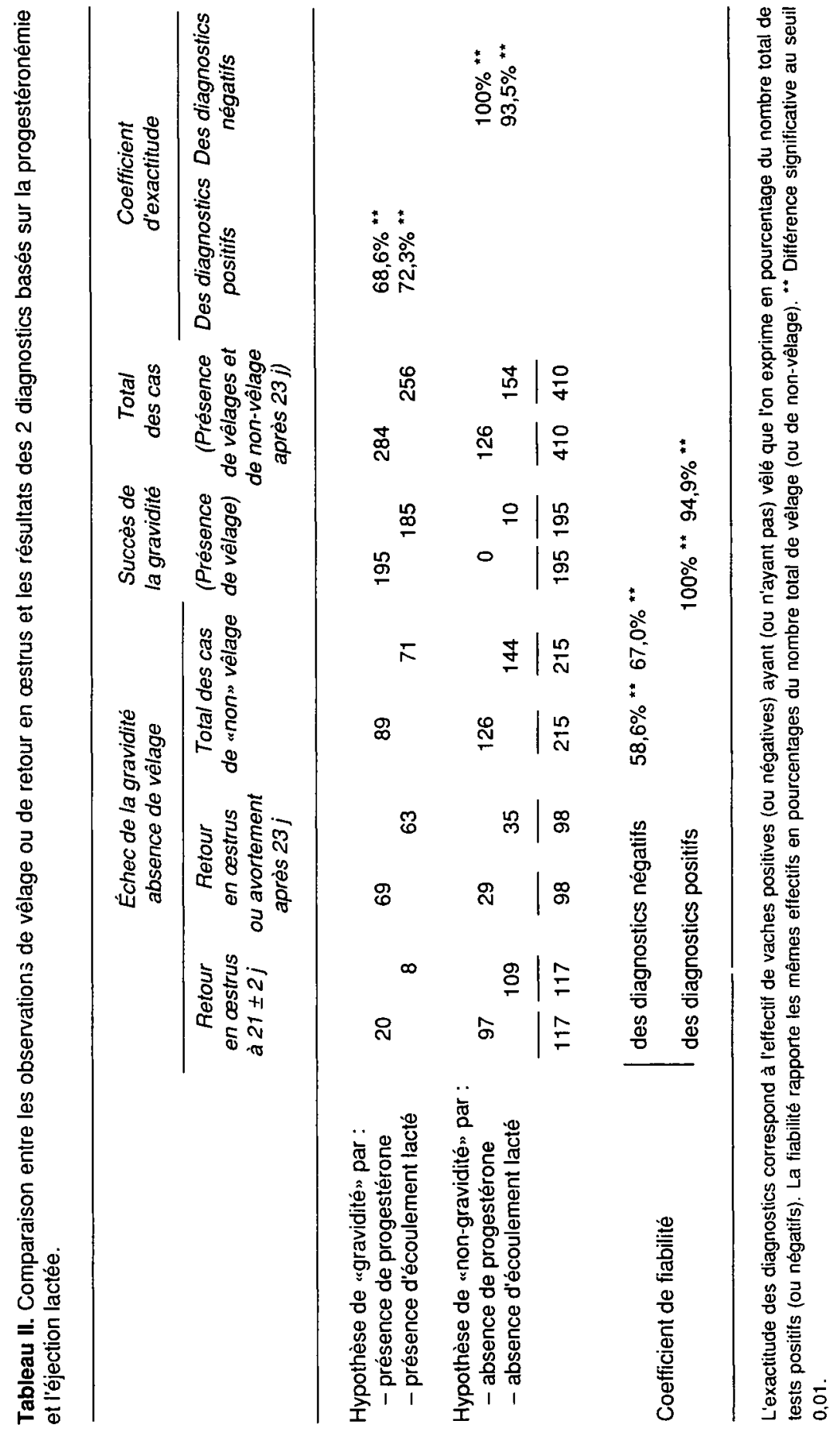




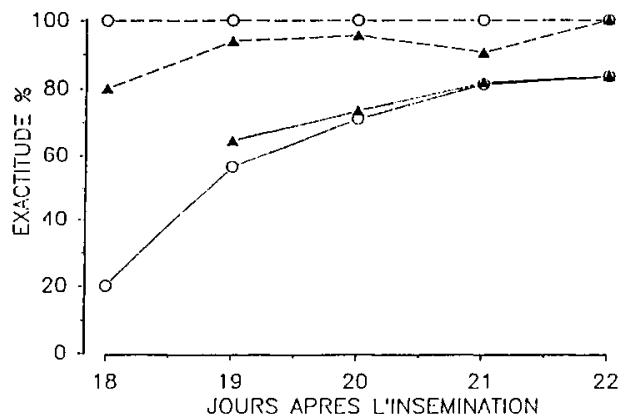

Fig 1. Exactitude des diagnostics positifs $(-)$ et négatifs $(---)$ des tests de gravidité basés sur la recherche de la progestérone plasmatique $(O)$ ou sur celle de l'évacuation du lait alvéolaire (A).

$E x_{d P}=\frac{V_{P V}}{V_{P}} \times 100 ; \quad E x_{d N}=\frac{V_{N n}}{V_{N}} \times 100$

$E x_{d P}=$ exactitude des tests positifs; $V_{P_{V}}=$ vaches positives ayant vêlé; $V_{P}=$ total des vaches positives; $E x_{d N}=$ exactitude des test négatifs; $V_{N n}=$ vaches négatives n'ayant pas vêlé; $V_{N}=$ total des vaches négatives.

et se préparer à les réinséminer immédiatement dès la fin du cyle infertile. En effet :

- sur les 117 vaches qui vont revenir en œstrus avant $23 \mathrm{j}$, elle permet d'en prédire 109 contre 97 (93,2 vs 82,9\%; $P<0,01)$;

- sur les 98 vaches non vues en chaleur à $23 \mathrm{j}$ (et qui pourtant ne termineront pas leur gravidité), elle permet de mieux discerner les négatives (35 contre 29) bien que les pourcentages ainsi obtenus $(35,7$ vs $29,7 \%$ ) ne soient pas significativement différents.

\section{DISCUSSION}

Le développement des méthodes de dosage immuno-enzymatique de la progesté-

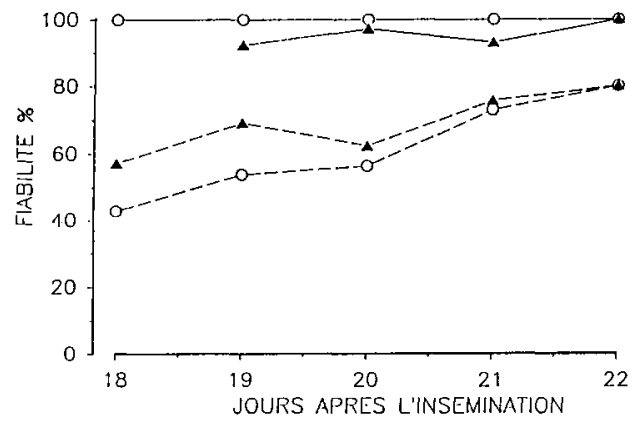

Fig 2. Fiabilité des diagnostics positifs (_- et négatifs $(---)$ des tests de gravidité basés sur la recherche de la progestérone plasmatique (O) ou sur celle de l'évacuation du lait alvéolaire (A).

$F_{d P}=\frac{V_{P V}}{V_{v}} \times 100 ; \quad F_{d N}=\frac{V_{N n}}{V_{n}} \times 100 ;$

$F_{d P}=$ fiabilité des diagnostics positifs; $V_{P_{V}}=$ vaches positives ayant vêlé; $V_{v}=$ total des vaches ayant vêlé; $F_{d N}=$ fiabilité des diagnostics négatifs; $V_{N n}=$ vaches négatives n'ayant pas vêlé; $V_{n}=$ total des vaches n'ayant pas vêlé.

rone a permis la diffusion commerciale de plusieurs kits de diagnostics, mais les éleveurs hésitent à les utiliser pour suivre l'activité ovarienne des vaches de leur troupeau, à cause de leur prix souvent élevé (Mialot, 1988) et de leur difficulté d'utilisation. Les réactifs ont une faible durée de conservation, les manipulations s'avèrent parfois longues et délicates; les étapes d'incubation et de lavage peuvent durer entre 10 et $30 \mathrm{~min}$ et la lecture, basée sur des changements colorimétriques (difficiles à codifier), également une dizaine de min (Davies et al, 1987; Thibier, 1988; Tainturier et al, 1988).

La technique que nous proposons ne présente pas ces inconvénients. D'une part, elle est peu onéreuse et ne fait appel 
qu'à des doses infimes de Dinolytic (256 $\mu \mathrm{g})$ (Labussière et al, 1988). D'autre part, son interprétation n'est pas ambigüe (le lait coule ou ne coule pas) et sa réalisation rapide (généralement moins de $5 \mathrm{~min}$ ). En effet, dès que la canule est introduite dans le canal d'un des trayons avant, il faut en moyenne un peu moins de 2 min (maximum $10 \mathrm{~min}$ ) pour évacuer les 23 ) $\mathrm{ml}$ de lait citernal (maximum $1620 \mathrm{ml}$ ). L'injection de PGF $2 \alpha$ peut alors avoir lieu et, en cas de maintien du corps jaune, il faut à nouveau attendre environ $1 \mathrm{~min} 30 \mathrm{~s}$ (maximum $6 \mathrm{~min}$ ) pour observer l'expulsion du lait alvéolaire induite par la libération d'ocytocine lutéale.

L'intérêt principal du test d'écoulement lacté repose sur sa précocité puisque son exactitude et sa fiabilité autorisent son utilisation dès le $20 \mathrm{e}$ j après l'insémination, soit 24-72 h plus tôt qu'en cas de recherche de la progestérone. Lorsque le diagnostic se révèle négatif, ceci permet de renouveler la tentative de fécondation sans attendre la fin du cycle suivant, comme c'est le cas lorsque les contrôles sont réalisés après le $21^{\mathrm{e}} \mathrm{j}$ (parfois au J23 à J24). Il est en effet recommandé de respecter ces délais lorsque les diagnostics sont basés sur la recherche de la progestérone (Thibier, 1988) puisque Humblot et al (1987) détectent 2 fois plus de vaches vides à J21 ou J23 qu'à J19. Ceci s'explique probablement par le fait qu'au $19^{\mathrm{e}} \mathrm{j}$, de nombreuses vaches non gestantes peuvent encore présenter une progestéronémie importante (Delahaut et al, 1978; Humblot et Dalla Porta, 1984) alors que la baisse d'ocytocine lutéale est plus précoce (Sheldrick et Flint, 1981 et 1983; Schams et Lahlou-Kassi, 1984; Wathes et al, 1984; Ivell et al, 1985; Abdelgadir et al, 1987; Jones et Flint, 1988; Marnet et Labussière, 1991, non publié).

Le nouveau test permet donc d'avancer les contrôles de 1 à 2 jours.
Nos résultats, acquis en moyenne avant $20 \mathrm{j}$, montrent, en effet, que le test d'écoulement est légèrement supérieur au test progestérone pour prédire les femelles qui vont revenir en chaleur avant 23 j (93,2\% vs 82.9) ou pour repérer celles, qui bien que non gravides, ne manifestent aucun œstrus avant ce stade $(35,7$ vs $29,7 \%)$. Elles pourraient alors être réinséminées sans risques (Thibier, 1988).

La fiabilité des résultats négatifs (rapportée aux mises-bas), est significativement supérieure avec notre méthode $(67,0$ vs $58,6 \%$ ) et elle aurait pu être améliorée si nous avions évité les contrôles prématurés $\mathrm{du} 18^{\mathrm{e}} \mathrm{j}$ qui ont conduit à un écoulement de lait sur 8 des 117 vaches revenant en cestrus avant 23 j (reliquats d'ocytocine sur quelques corps jaunes en fin de lutéolyse).

A contrario, l'absence d'éjection lactée au cours des 4 premières min qui suivent l'administration de PGF2 $\alpha$ ne constitue pas une preuve absolue de gravidité, puisque, sur les 195 vaches dont la gestation s'est terminée par un vêlage, nous en avons dénombré 10 qui n'ont pas eu d'écoulement et il est permis de supposer que ces quelques cas résultent d'une erreur d'injection de PGF2 $\alpha$ hors de la veine mammaire.

Malgré ces réserves, il importe de souligner 3 points relatifs à l'exactitude des résultats positifs obtenue avec le test d'écoulement :

- elle est significativement supérieure $(72,3 \%)$ à celle fournie par la progestéronémie $(68,6 \%)$. Pour cette méthode, la discrimination aurait toutefois pu être plus efficace si nous avions placé le seuil de présomption de gravidité au-dessus de 1,5 $\mathrm{ng} / \mathrm{ml}$ de progestérone. En effet, les 195 cas de vêlage présentent tous des taux supérieurs à $2,4 \mathrm{ng} / \mathrm{ml}$ et $92,5 \%$ d'entre eux se situent même au-dessus de $4,8 \mathrm{ng} / \mathrm{ml}$. On peut encore affiner la caractérisation des futures parturientes en adjoignant à 
ces valeurs celle du lait alvéolaire recueilli après administration de PGF2 $\alpha$; dans ces conditio 1 , $97 \%$ des vaches gravides jusqu'à terme dépassent à la fois $4,8 \mathrm{ng} / \mathrm{ml}$ et $1200 \mathrm{ml}$ de lait expulsé;

- elle atteint déjà $73,2 \%$ dès le $20^{\circ}$ jour, ce qui correspond à l'ordre de grandeur des valeurs fournies jusqu'à maintenant à des stades plus tardifs (J21 à J23) par les dosages RIA ou EIA de progestérone (Hoffman et al, 1976; Cox et al, 1978; Ball, 1978; Kummerfeld et al, 1982; Sauer et al, 1986; Stanley et al, 1986; Van de Wiel et Koops, 1986; Humblot et al, 1987; Thibier, 1988).

Cette exactitude de $73,2 \%$ est d'ailleurs sous-estimée par rapport aux pourcentages publiés par certains de ces auteurs qui ont minimisé leurs risques d'erreurs en ne prenant en compte que les contrôles effectués avant un retour en chaleur, puisque, dans les conditions de terrain, l'éleveur n'aurait pratiqué le test que sur les vaches non encore vues en cstrus;

- elle n'est pas aussi bonne que celle obtenue, i'n peu plus tardivement ( $\left.30^{\mathrm{e}} \mathrm{j}\right)$, par dosage de la PSPB; Humblot (1991) rapporte alors des valeurs voisines de $90 \%$ qui font de cette méthode, basée sur la recherche d'un signal embryonnaire, une confirmation des gestations présumées par les sécrétions lutéales de progestérone ou d'ocytocine.

\section{CONCLUSION}

Dès le $20^{e} \mathrm{j}$ après l'insémination, il est possible en 5-10 min et d'une façon facile à codifier (présence ou non d'un écoulement de lait) d'aboutir à une exactitude satisfaisante des diagnostics de gestation positifs et de détecter les femelles vides avec une bonne fiabilité. II ne faut pas sous-estimer les risques d'erreurs rencon- trés chez les vaches qui, bien que gravides, présentent une réponse négative au test d'éjection (10 sur 195); l'inexpérience du manipulateur peut à cet égard être primordiale (injection intraveineuse de PGF2 $\alpha$ non réussie, absence de lait dans la lumière des alvéoles consécutive à une décharge prématurée d'ocytocine lors de la désinfection des trayons). Nos études antérieures sur les modalités de réponses aux prostaglandines pendant les 2 premiers mois qui suivent l'insémination (Labussière et al, 1988) ainsi que l'inocuité et le faible coût de la méthode, autorisent son utilisation répétée pour confirmer ou infirmer les premiers résultats éventuellement erronés.

Pour les mêmes raisons, on peut envisager d'utiliser cette méthodologie pendant la période post-partum pour rechercher les premières ovulations et la reprise de l'activité ovarienne; une expérimentation sur ce thème est en cours.

\section{REMERCIEMENTS}

Nous tenons à remercier Mme A Briand et l'ensemble du personnel animalier des troupeaux de vaches laitières de l'INRA (Rennes, Nouzilly et Bressonvilliers) pour l'aide précieuse qu'ils nous ont apportée lors de la réalisation de ce travail.

\section{RÉFÉRENCES}

Abdelgadir SE, Swanson LV, Oldfield JE, Stormshak F (1987) Prostaglandin F2 $\alpha$ induced release of oxytocin from bovine corpora lutea in vitro. Biol Reprod 37, 550-555

Ball PJH (1978) The relationship of age and stage of gestation to the evidence of embryo death in dairy cattle. Res Vet Sci 25, 120-122

Booth JM, Davies J, Holdsworth RJ (1979) Use of the milk progesterone test for pregnancy determination. Br Vet J 135, 478-488 
Chaffaux S, Bianchi M, Bhat P, Hedge GV, Reddy GNJ, Thibier M (1988) L'échographie en temps réel par voie transrectale. Intérêt pour le diagnostic de gestation chez la vache. Rec Med Vet 164, 101-108

Cox NG, Thompson FN, Culver DH (1978) Milk progesterone to predict reproductive status in a commercial dairy herd. J Dairy Sci 61, 1616-1621

Davies J, Fletcher NA, Newstead RA (1987) Evaluation of an enzyme immunoassay kit for the qualitative assessment of progesterone in bovine milk samples. Vet Rec 120, 206-207

Delahaut $P$, Beckers JF, Ectors F (1978) Diagnostic précoce de gestation chez les différentes espèces animales. Ann Med Vet 122 , 205-208

Heap RB, Holdsworth RJ, Gadsby JE, Laing JA, Walters DE (1976) Pregnancy diagnosis in the cow from milk progesterone concentration. Br Vet J 132, 445-464

Hoffman B, Gunzler O, Hamburger R, Schmidt W (1976) Milk progesterone as a parameter for fertility control in cattle, methodological approach and present status of application in Germany. Br Vet J 132, 469-477

Humblot $P$ (1991) Signaux embryonnaires et contrôle de la gestation des ruminants. Rec Med Vet 67, 193-202

Humblot P, Dalla Porta (1984) Effect of conceptus removal and intra uterine administration of conceptus tissue on luteal function in the cow. Reprod Nutr Dev 24, 529-541

Humblot $P$, Thibier M (1984) Evaluation comparée des méthodes de diagnostic de gestation chez les bovins. Elev Insem 200, 3-18

Humblot $P$, Magnin $M$, Thibier $M$, Somme $G$ (1987) Diagnostic précoce de non-gestation à la ferme chez les bovins; choix du meilleur jour de prélèvement du lait. Elev Insem 219 , 15-22

Humblot P, Camous S, Martal J, Charlery J, Jeanguyot $N$, Thibier $M$, Sasser $G$ (1988) Diagnosis of pregnancy by radioimmunoassay of a pregnancy specific protein in the plasma of dairy cows. Theriogenoloy 30 , 193-202

Ivell R, Brackett KH, Fields MJ, Richter D (1985) Ovulation triggers oxytocin gene expression in the bovine ovary. FEBS 190, 263-267
Jones DS, Flint AP (1988) Concentrations of oxytocin-neurophysin prohormone mRNA in corpora lutea of sheep during the oestrus cycle in early pregnancy. $J$ Endocrinol 117 , 409-414

Kishimoto Y, Kato H, Mitani M (1987) Enzyme immunoassays of progesterone in bovine plasma and skim milk and its application to early pregnancy diagnosis. $J J p n$ Vet Med Assoc 40, 161-164

Kummerfeld $\mathrm{HL}$, Oltenacu EAB, Foote $\mathrm{RH}$ (1982) Embryonic mortality in dairy cows estimated by non-return to service, oestrus and cyclic milk progesterone patterns. J Dairy Sci 61, 1773-1777

Labussière $\mathrm{J}$, Philibert $\mathrm{C}$, Combaud JF, Dotchewski D (1982) Étude de l'efficacité de PGF2 $\alpha$ sur l'éjection du lait au cours du cycle sexuel de la vache. Reprod Nutr Dév 22, 4963

Labussière $\mathrm{J}$, Thomas $\mathrm{Ph}$, Combaud $\mathrm{JF}$, de la Chevalerie FA (1988) Éjection du lait induite par PGF2 $\alpha$ pendant les deux premiers mois qui suivent l'insémination des vaches. Applications à un diagnostic de gravidité. Reprod Nutr Dév 28, 899-907

Labussière $\mathrm{J}$, Lacroix MC, Combaud JF, de la Chevalerie FA, Thomas P (1990) Suppression par la lutectomie de l'accroissement simultané de la pression intramammaire et de l'ocytocinémie induit par l'injection de PGF2 $\alpha$ chez la brebis. Reprod Nutr Dev 30, 91-96

Laing JA, Heap RB (1973) The concentration of progesterone in the milk of cows during the reproductive cycle. Br Vet J 127, 19-22

Laitinen J, Remes E, Tenhunen M, Hanninen O, Alanko M (1985) Milk progesterone in Finnish dairy cows, a field study on the control of artificial insemination and early pregnancy. $\mathrm{Br}$ Vet $J 141,297-307$

Mialot JP (1988) Comparaison de kits disponibles. Bull Tech Insem Artif 49, 42-44

Sasser RG, Ruder CA, Ivani KA, Butler JE, Hamilton WC (1986) Detection of pregnancy by radioimmunoassay of a novel pregnancy specific protein in serum of cows and a profile of serum concentrations during gestation. Biol Reprod 35, 936-942

Sauer JM, Foulkes JA, Worsiold A, Morris BA (1986) Use of progesterone 11-glucuronidealkaline phosphate conjugate in a sensitive 
microtitre plate enzyme immuno assay of progesterone in milk and its application to pregnancy testing in dairy cattle. $J$ Reprod Fertil 76, 375-391

Saumande J, Tamboura S, Chupin D (1985) Changes in milk and plasma concentrations of progesterone in cows after treatment to induce superovulation and their relationships with number of ovulations and of embryos collected. Theriogenology 23, 719-731

Schams D, Lahlou-Kassi A (1984) Circulating concentrations of oxytocin during pregnancy in ewes. Acta Endocrinol 106, 277-281

Schwartz D (1963) Méthodes statistiques à l'usage des médecins et des biologistes. Flammarion Médecine - Sciences, Paris, 5565

Sheldrick EL, Flint APF (1981) Circulating concentrations of oxytocin during the oestrus cycle and early pregnancy in sheep. Prostaglandins 22, 631-636

Sheldrick EL, Flint APF (1983) Luteal concentration of oxytocin decline during early pregnancy in the ewes. J Reprod Fertil 68, 477-480

Stanley CF, Paris F, Webb AE, Heap RB, Ellis ST, Hamon M, Worsfold A, Booth JM (1986) Use of a new and rapid milk progesterone assay to monitor reproduction activity in the cow. Vet Rec 118, 664-667

Tainturier D, André F, Chaari M, Sardjana KW, Le Net JL, Lijour L (1983) Intérêt de l'échotomographie pour le contrôle de la reproduction d'un grand troupeau de vaches laitières. Rev Med Vet 134, 419-424

Tainturier D, André F, Fieni F (1988) Diagnostic précoce de la non-gestation chez la vache par un dosage qualitatif de la progestérone.
Comparaison de trois tests ELISA rapides. Rev Med Vet 139, 1115-1118

Tamboura D (1983) Les concentrations de progestérone dans le lait de vache. Mise au point d'une technique de dosage. Relations avec les réponses de l'ovaire à un traitement de superovulation. Thèse Doct Ingen ENSA Montpellier, $90 \mathrm{p}$

Terqui M, Thimonier J (1974) Nouvelle méthode radio-immunologique rapide pour l'estimation du niveau de progestérone plasmatique. Application pour le diagnostic précoce de gestation chez la brebis et la chèvre. CR Séances Acad Sci Paris Sér D 279, 1109-1112

Terqui M, André D (1982) Quand savoir si ma vache est en gestation : 3 semaines après l'insémination ? Elev Bovin 115, 93-99

Thibier M (1980) Le diagnostic précoce de gestation et la mesure de la progestérone dans le lait. Elev Insem 180, 9-14

Thibier M (1988) Stéroïdes sexuels et diagnostics de gestation chez les bovins. Tests en ferme. Reprod Nutr Dév 28, 1747-1752

Van de Wiel DFM, Koops WK (1986) Development and validation of an enzyme immuno assay for progesterone in bovine milk or blood plasma. Anim Reprod Sci 10, 201-213

Wathes DC, Swann RW, Pickering BT (1984) Variations in oxytocin, vasopressin and neurophysin concentrations in the bovine ovary during the oestrus cycle and pregnancy. J Reprod Fertil 71, 551-557

Wathes DC, Swann RW, Porter DG, Pickering BT (1986) Oxytocin as an ovarian hormone. In: Neurobiology of oxytocin. Current topics. Springer-Verlag, Berlin-Heidelberg. NeuroEndocrinology 6, 129-152 